\title{
Small Auxin Up RNAs influence the distribution of indole-3-acetic acid and play a potential role in increasing seed size in Euryale ferox Salisb
}

Zhiheng Huang ${ }^{1,2}$, Ke Bao $^{1,2}$, Zonghui Jing ${ }^{1,2}$, Qian Wang ${ }^{1,2}$, Huifang Duan ${ }^{1,2}$, Yaying Zhu ${ }^{1,2}$, Sen Zhang ${ }^{1,2}$ and Qinan $\mathrm{Wu}^{1,2^{*}}$

\begin{abstract}
Background: Aquatic Euryale ferox Salisb. is an economically important crop in China and India. Unfortunately, low yield limitations seriously hinder market growth. Unveiling the control of seed size is of remarkable importance in improvement of crops. Here, we generated a new hybrid line $(\mathrm{HL})$ with larger seeds by crossing South Gordon Euryale and North Gordon Euryale (WT) which hasn't been reported before. However, the functional genes and molecular mechanisms controlling the seed size in Euryale ferox Salisb. remain unclear. In this study, we focused on the differentially expressed genes in the auxin signal transduction pathway during fruit development between $\mathrm{HL}$ and WT to explore candidate regulatory genes participated in regulating seed size.

Results: Both concentration and localization of indole-3-acetic acid (IAA) at two growth stages of fruits of WT and HL were detected by LC-MS and immunofluorescence. Although IAA content between the two lines did not differ, IAA distribution was significantly different. To elucidate the mechanism and to seek the key genes underlying this difference, RNA-seq was performed on young fruits at the two selected growth stages, and differentially expressed genes related to the auxin transduction pathway were selected for further analysis.

Conclusion: Hybrid Euryale ferox Salisb. expressed significant heterosis, resulting in non-prickly, thin-coated, large seeds, which accounted for the significantly larger yield of HL than that of WT. Our study indicated that Small Auxin Up RNAs (SAURs) -mediated localization of IAA regulates seed size in Euryale ferox Salisb. We found that some SAURs may act as a positive mediator of the auxin transduction pathway, thereby contributing to the observed heterosis.
\end{abstract}

Keywords: Euryale ferox Salisb, Hybrid, RNA-seq, IAA, SAUR

\footnotetext{
* Correspondence: qnwyjs@126.com

${ }^{1}$ School of Pharmacy, Nanjing University of Chinese Medicine, 138 xianlin Road, Nanjing 210023, Jiangsu, China

${ }^{2}$ Jiangsu Collaborative Innovation Center of Chinese Medicinal Resources

Industrialization, Nanjing 210023, China
}

C The Author(s). 2020 Open Access This article is licensed under a Creative Commons Attribution 4.0 International License, which permits use, sharing, adaptation, distribution and reproduction in any medium or format, as long as you give appropriate credit to the original author(s) and the source, provide a link to the Creative Commons licence, and indicate if changes were made. The images or other third party material in this article are included in the article's Creative Commons licence, unless indicated otherwise in a credit line to the material. If material is not included in the article's Creative Commons licence and your intended use is not permitted by statutory regulation or exceeds the permitted use, you will need to obtain permission directly from the copyright holder. To view a copy of this licence, visit http://creativecommons.org/licenses/by/4.0/ The Creative Commons Public Domain Dedication waiver (http://creativecommons.org/publicdomain/zero/1.0/) applies to the data made available in this article, unless otherwise stated in a credit line to the data. 


\section{Background}

Euryale semen, also called 'fox nut' and 'Qian shi' in mandarin, is the seed of Euryale ferox Salisb., an important food, ornamental, and medicinal species widely distributed in the southern region of China and North Bihar, India [1]. The species is often considered as an aquatic food due to its high starch content (more than $70 \%$ ); additionally, in some areas, people prefer Euryale seed to rice for meal because of its low glycemic index [2]. Furthermore, Euryale semen is also a common Chinese traditional medicine whose pharmacological properties including, anti-depression, anti-oxidant, and antidiabetic action, have been extensively demonstrated by numerous studies [3-5].

Euryale ferox Salisb. can be divided into two main types: North Gordon Euryale (wildtype or WT) and South Gordon Euryale (SE) type, as shown in Fig. 1. The WT is distributed in most areas, and bears small, prickly, thin-coated seeds, whereas SE, mainly cultured in Jiangsu and Anhui provinces, bears non-prickly, thickcoated large grains [6]. The yield of the WT is approximately three times higher than that of SE, largely because of the low pod-filling that characterizes SE. However, seeds of SE are tastier than those of the WT, which suggests a starch structural difference between the two types. Therefore, in order to obtain a high yielding crop with tasty seeds, we crossed WT and SE in 2015, and a hybrid line (HL) was successfully developed after self-crossing for three generations. The HL produces thornless tissues and large seeds, which are much more in common with the characteristics of SE, but its growth pattern is more similar to WT (Fig. 1c, f and i). Externally, floating leaves (peltate, $>1.8 \mathrm{~m}$ diameter) of HL are green, non-prickly above and red or purple, thorny beneath. Flowers are solitary with four persistent and non-prickly sepals, together with several purple petals. The inferior ovary, below each flower, develops into a spongy berry-like fruit which is non-prickly, and each mature fruit contains 60-90 seeds with thin black seed coats. HL is advantageous for having a higher yield, with the average yield of HL in experimental plots up to $4875 \mathrm{~kg} \mathrm{ha}^{-1}, 35 \%$ greater than the yield of the WT in
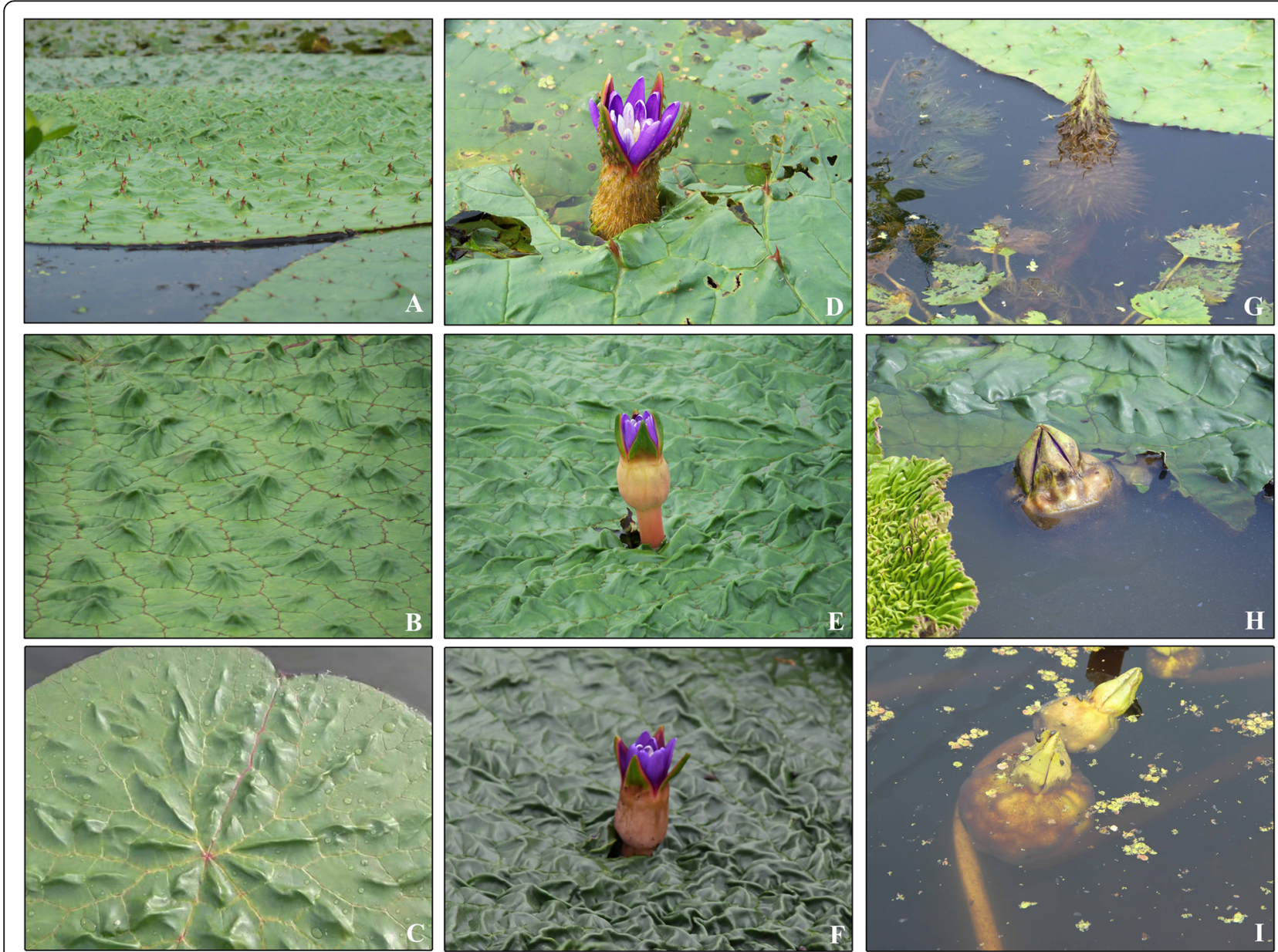

Fig. 1 Appearance of WT and SE. The WT has prickly leaves (a), flowers (d) and fruits (g), whereas the SE generates non-prickly leaves (b), flowers (e) and fruits (h). The HL generation has non-pricky leaves (c), flowers (f) and fruits (i) similar to that of SE 
2018. However, the seed number per plant did not show any difference between the WT and HL, indicating the yield evaluation in HL was mainly attributed to the size and weight of seeds. In a previous study, we did not detect any difference between WT and SE at the DNA level, which suggested both types belong to the same species, whereby transcriptional differences must be the cause for the phenotypic differences between them.

Endogenous phytohormones are well known to play critical roles in plant physiological and biochemical processes at the molecular level [7-9]. Among plant growth regulators, auxin (indole-3-acetic acid, IAA) regulates whole plant developmental processes $[10,11]$. IAA affects cell division and cell elongation; furthermore, some yield traits in all higher plants are largely dependent on this small organic-acid molecule [12]. Plant architecture has been demonstrated to be directly related to yield and to be controlled by pin-formed5b (PIN5b) through changes in IAA homeostasis, transport, and distribution [13]. Further, these roles of IAA have been demonstrated in many genetic studies on the regulation of IAA through control of the metabolism of IAA, changes of IAA distribution pattern and on plant responses to IAA signaling $[14,15]$. Furthermore, it is increasingly interesting to investigate the role of genes included in IAAsignal transduction pathway in biological process mediated by auxin [16]. Up to present, a large number of IAA-related factors included in IAA-signal transduction pathway have been documented in plants, including the F-box TRANSPORT INHIBITOR RESPONSE 1/AUXIN SIGNALING F-BOX PROTEIN (TIR1/AFB) auxin coreceptors, the AUXIN RESPONSE FACTOR (ARF) transcription factors, and the Auxin/INDOLE-3-ACETIC ACID (Aux/IAA) transcriptional repressors [17, 18]. Auxin stimulates the interaction between TIR1/AFB and Aux/IAA proteins and then induces the degradation of Aux/IAA and the release of ARF factors, thereby contributing to the rapid transcription of a set of auxinresponsive genes which implicate in diverse processes in plants, such as embryogenesis and organogenesis [19, 20]. Thus, the Small Auxin Up RNA (SAUR), Glycoside Hydrolase 3 (GH3) and AUX/IAA genes are three primary auxin-responsive gene families; unfortunately, functional studies on SAURs have lagged behind [21] The reported genetic functions of SAURs include elongating soybean hypocotyl sections and promoting Arabidopsis hypocotyl and stamen filament elongation, which indicate a role for SAURs in the regulation of cell expansion [22-24]. In a previous study, OsSAUR39 was shown to influence rice yield through mediating auxin synthesis and transport, implying a potential ability of SAURs to regulate crop yield [25-27]. It's not clear whether the SAURs and IAA-signal transduction pathway is related with the increased seed size in HL.
Due to the distribution of WT is much broader than the distribution of SE in China and India, and most studies investigated formerly used WT as experimental material, only WT was thereby employed as a wild-type control in this study [28, 29]. To explore the potential functional genes for the promotion of seed size in Euryale ferox Salisb., we subjectively divided growth into stages I through $\mathrm{V}$ according to fruit growth regulation in Euryale ferox Salisb., after observing largely difference in phenotype and IAA distribution between WT and HL. Then, RNA-seq was performed on fruits at stages II and III to identify the differentially expressed genes (DEGs) involved in the IAA signal transduction pathway. These results indicated that SAURs-mediated localization of IAA regulates seed size in Euryale ferox Salisb.

\section{Results}

\section{$\mathrm{HL}$ produces larger seeds and kernels}

After growing for 21 weeks, the two plant types matured and their seeds were collected. It is worth noting that the seed number per plant did not show any difference between the two lines in our data (on average, WT was about 1170 seeds per plant, while HL was 1180), which indicated that the yield difference between these two lines was probably attributed to seed size and weight. Although the weight per seed of HL (range from $1.1 \mathrm{~g}$ to $1.7 \mathrm{~g}$ ) was much heavier than that of WT (range from $0.5 \mathrm{~g}$ to $0.9 \mathrm{~g}$ ), we paid more attention to seed size than to weight because, during the growth of reproductive, weight gain is most noticeable in the rapid changes in seed size [30]. All seeds were subjected to a brief drying period in the sun, and then seed size and kernel number of WT and HL were recorded. As shown in Fig. 2, HL (B) produced larger kernels than WT (A). As Fig. 2c shows, although the seed coat of HL was obviously thicker, the large size of the seeds contributed to larger kernels in HL.

\section{The difference in fruit length between the two experimental lines became significant at growth stage III}

To further understand the growth regulation of Euryale ferox Salisb., we divided development into five stages (I through V), as in rice studies [31, 32]. At stage I, fruits grow at a slow pace; at stage II, no significant growth in fruit size has occurred; at stage III, fruits show fast growth; at stage IV, fruits are nearly mature, and, at stage $\mathrm{V}$, fruits have entered post-maturity (Fig. S1). We collected all fruits on the 10th week according to the growth pattern, and, more importantly, fruit growth at this phase contributed to the differences between the two lines later in development. As shown in Fig. 3, fruit length in HL was greater than in WT at stages III $(p<0.05)$, consequently, we selected samples at stages II and III (fruit width can be found in Fig. S2). Further, both HL and WT produced 12 fruits above water level per week. 


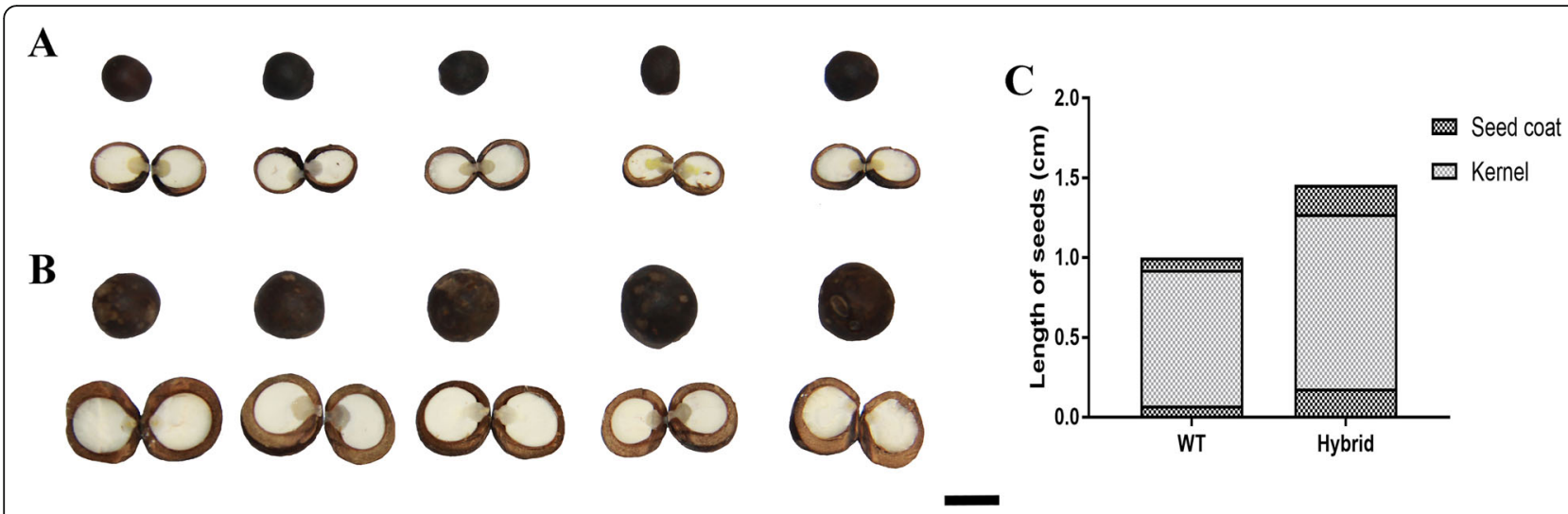

Fig. 2 The seed size of WT and HL was measured. The obviously differences between WT (a) and HL (b) were observed in size of seeds, kernels and coats $(\mathrm{bar}=1 \mathrm{~cm})$. And $(\mathbf{c})$ length of both kernel and coat in seed of HL are significantly larger than in WT

Localization of IAA in HL differed from that in WT

Phytohormones play important roles in both plant growth and crop yield. IAA is one of the most active effectors in young tissues [33]. We measured IAA content at the initial growth stages and found no difference between fruits of the two lines at stage III (Fig. 4a). However, both concentration and localization of IAA at the cellular level, which must control cell activity and/or its fate, are critical [34]. Therefore, to investigate the localization of IAA in fruits at the same stage, immunofluorescence staining was performed on sectioned fruits. Surprisingly, at stage III, WT allocated free-IAA to its special tissue, pricks, more frequently, whereas the fluorescence signal in fruits of HL concentrated in the ovary and the stamen (Fig. 4b). Similar results were found at stage II (Fig. S3), and the negative control could be
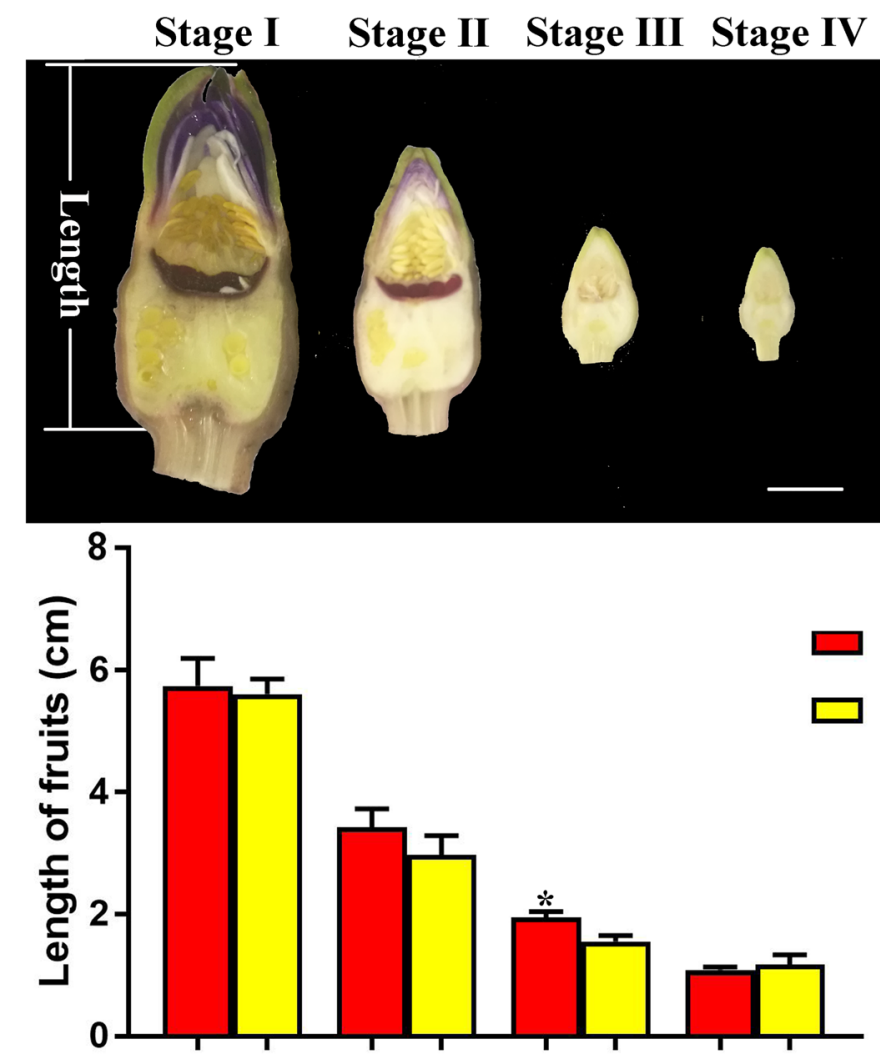

Fig. 3 On the 10th week, the length of fruits at different stages was measured. Fruits in $\mathrm{HL}$ (top of the Fig. 3) grew significantly faster than in WT at stage III among four stages. ${ }^{*} P<0.05$, compared to WT (bar $\left.=1 \mathrm{~cm}\right)$ 

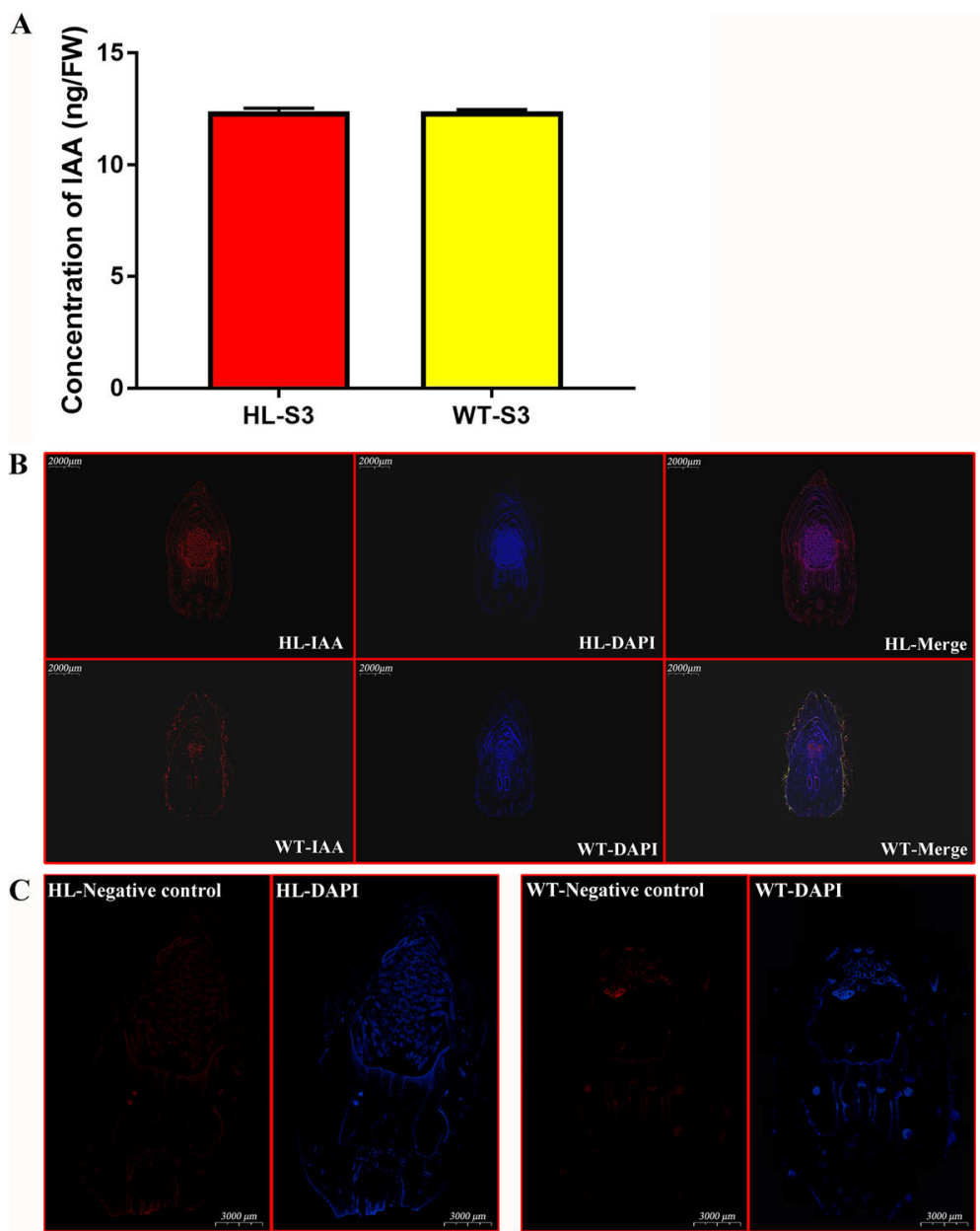

Fig. 4 Concentration and distribution of IAA in fruits at stage III were detected by LC-MS and immunofluorescence. (a) Fruits in HL and WT did not show significant difference in concentration of IAA at stage II, while (b) the localization of IAA (red) in WT was partly distributed in prick. S3, stage III. (c) Negative control

found in Fig. 4c. This most interesting finding suggested a potential role for auxin signal response in regulating ovary size and, subsequently, seed size. We also detected the localization of free-IAA signal in SE fruits with similar size (Fig. S4). It was found that free-IAA only located in stamens of SE fruit, indicating different utilizations of IAA among three types. However, it's also possible that SE fruit (even if with similar size) was at later growth stage, because the fruit of SE grows much faster than that of WT and HL.

\section{RNA-seq and transcriptome assembly}

A series of de novo assemblies were carried out with Trinity. To obtain useful transcriptomic information for the two lines during development, $12 \mathrm{cDNA}$ libraries were established and paired-end sequence reads were generated using the Illumina Hiseq X10. As shown in Table 1, a total of $630,221,090$ clean reads were generated from HL and WT cDNA libraries. The average base fraction with quality scores of Q30 and Q20 were 94.6 and $98.1 \%$, which indicated high quality data. Relative length of assembled sequences is an important evaluation criterion for assembly quality, and the summary statistics of the assembled clean reads are shown in Table 2. Using the Trinity assembler, 197,253 unigenes were generated with an average length of $959 \mathrm{nt}$, an N50 of $1574 \mathrm{nt}$, and a total length of 189,170,382 nt. Total unigenes included 44,511 (22.57\%) of less than $300 \mathrm{nt}$, $151,012(76.55 \%)$ with lengths from 301 to $5000 \mathrm{nt}$, and $1730(0.88 \%)$ with lengths greater than $5000 \mathrm{nt}$ These results proved high quality sequencing and assembly in our samples.

\section{Annotation and GO classification}

The annotation of the 197,253 assembled unigenes revealed that 44,363 (22.49\%), 18,229 (9.24\%), 26,746 (13.56\%), 9633 (4.88\%), 3632 (1.84\%), and 2879 (1.46\%) showed significant similarity in the GO, Pathway Pfam, 
Table 1 Statistical Analysis of Transcriptome Sequencing Data

\begin{tabular}{|c|c|c|c|c|c|}
\hline Sample & Clean reads number & Clean bases & Clean rate(\%) & Q20(\%) & Q30(\%) \\
\hline WT-S2-1 & $49,343,466$ & $7,392,663,824$ & 95.64 & 97.78 & 93.69 \\
\hline WT-S2-2 & $35,660,154$ & $5,349,023,100$ & 95.3 & 98.03 & 94.77 \\
\hline WT-S2-3 & $46,271,622$ & $6,946,934,906$ & 94.97 & 97.84 & 93.84 \\
\hline WT-S3-1 & $38,688,882$ & $5,803,332,300$ & 95.66 & 98.11 & 94.98 \\
\hline WT-S3-2 & $53,603,992$ & $8,023,343,356$ & 97.29 & 97.69 & 93.01 \\
\hline WT-S3-3 & $65,400,866$ & $9,791,552,137$ & 96.21 & 97.77 & 93.57 \\
\hline HL-S2-1 & $106,884,270$ & $16,025,803,643$ & 97.05 & 97.96 & 93.96 \\
\hline HL-S2-2 & $31,283,988$ & $4,692,598,200$ & 95.41 & 98.07 & 94.89 \\
\hline HL-S2-3 & $48,644,712$ & $7,296,706,800$ & 96.35 & 98.42 & 95.62 \\
\hline HL-S3-1 & $42,801,744$ & $6,420,261,600$ & 96.21 & 98.4 & 95.59 \\
\hline HL-S3-2 & $40,785,442$ & $6,117,816,300$ & 96.05 & 98.43 & 95.66 \\
\hline $\mathrm{HL}-\mathrm{S} 3-3$ & $46,852,120$ & $7,027,818,000$ & 96.41 & 98.43 & 95.66 \\
\hline
\end{tabular}

TMhmm, Eggnog, SignalP, and Uniprot databases, respectively. In all, 71,399 unigenes $(36.20 \%$ of all unigenes) were successfully annotated in at least one database (Table 3). As shown in Fig. 5, total 44,363 unigenes annotated in $\mathrm{GO}$ were assigned to three principal domains; "cellular component", "molecular function", and "biological process".

\section{SAURs induced differences in the IAA signal transduction}

\section{pathway between HL and WT}

The KEGG Pathway database records the networks of molecular interactions in cells and species-specific variations [35]. According to the findings on localization of IAA reported above, we focused on genes involved in

Table 2 Length distribution of the unigenes in transcriptome

\begin{tabular}{llc}
\hline Length range & Unigenes & Percentage (\%) \\
\hline $200-300$ & 44,511 & 22.57 \\
$300-500$ & 43,507 & 22.06 \\
$500-1000$ & 48,729 & 24.70 \\
$1000-2000$ & 36,800 & 18.66 \\
$2000-5000$ & 21,976 & 11.14 \\
$5000+$ & 1730 & 0.88 \\
Total number & 197,253 & \\
Total length & $189,170,382$ & \\
N40 length & 1980 & \\
N50 length & 1574 & \\
N60 length & 1220 & \\
N70 length & 910 & \\
N80 length & 633 & \\
N90 length & 390 & \\
Min length & 201 & \\
Max length & 17,279 \\
Mean length & 959 & \\
\hline
\end{tabular}

the auxin transduction pathway. The pathway is shown in Fig. 6a; briefly, auxin promotes an interaction between TIR1/AFB and Aux/IAA proteins, resulting in the degradation of the Aux/IAAs and the release of ARF repression, and down-stream SAURs, GH3 and AUX/IAA are activated subsequently. We compared the transcriptome from HL with that from WT at both stages II and III: only SAURs showed a significant difference in the whole pathway (Fig. 6a). Then, all unigenes annotated with $A R F, A U X / I A A, G H 3$ or SAUR were selected for comparison (Fig. 6b). An obvious difference was observed in SAURs, while the other three families did not show any changes between the two lines at either growth stage. It is worth noting that the unigene number of SAURs is the least among the four families, which indicated less functional redundancy in SAURs of Euryale ferox Salisb. Interestingly, this finding suggests an important role for SAURs in responding to IAA and, probably, upregulating seed size in HL.

\section{Verification of RNA-seq by RT-qPCR}

To evaluate the validity of the expression of DEGs, 10 candidate unigenes involved in the auxin transduction

Table 3 Unigenes annotated in databases

\begin{tabular}{lll}
\hline Database & Number of unigenes & Percentage (\%) \\
\hline GO & 44,363 & 22.49 \\
Pathway Pfam & 18,229 & 9.24 \\
TMhmm & 26,746 & 13.56 \\
Eggnog & 9633 & 4.88 \\
signalP & 3632 & 1.84 \\
Uniprot & 2879 & 1.46 \\
All database & 68,200 & 34.57 \\
At least one database & 71,399 & 36.2 \\
Total unigenes & 197,253 & 100 \\
\hline
\end{tabular}




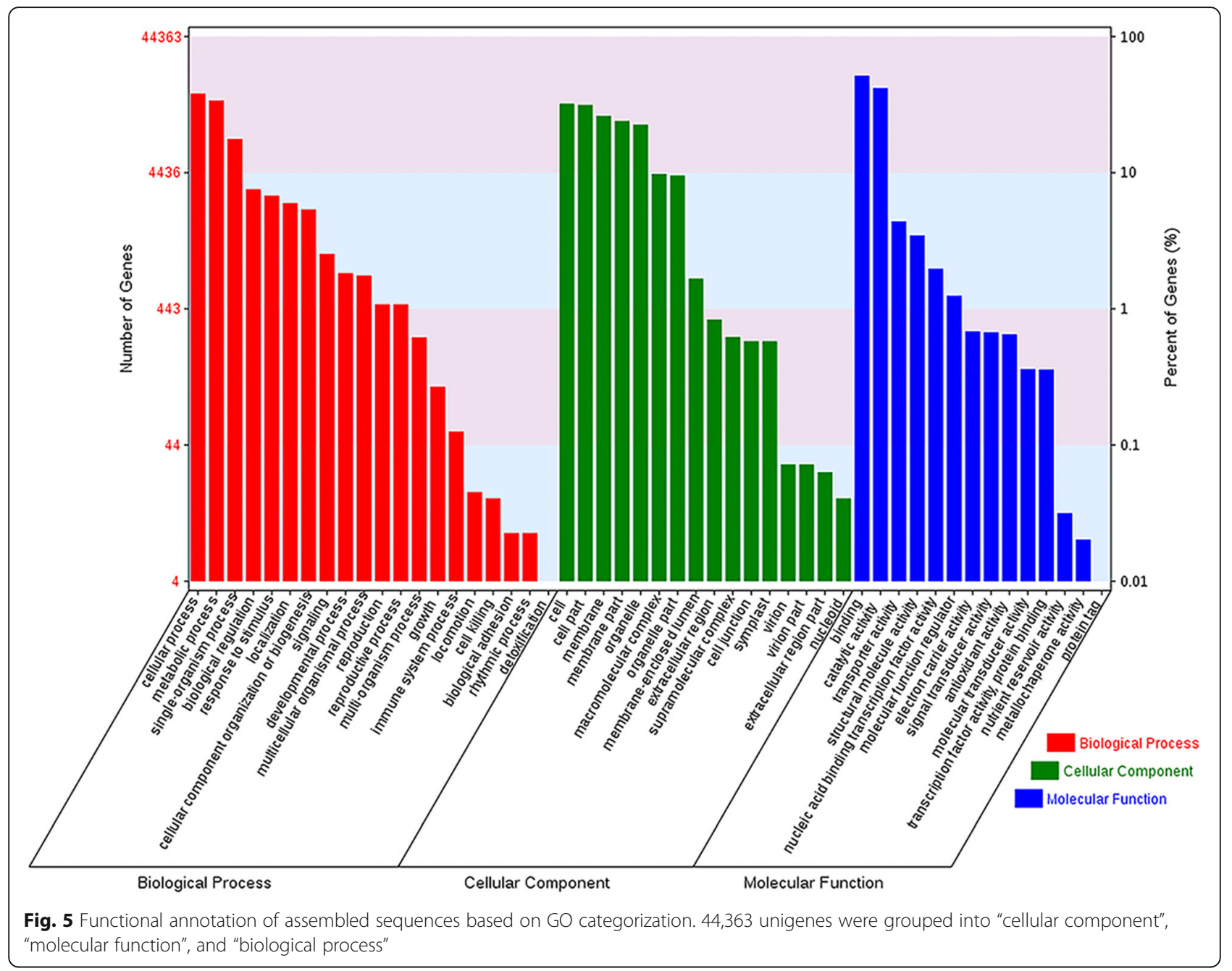

pathway (2-ARFs, 3-AUX/IAAs, 2-GH3s, and 4-SAURs) were detected by RT-qPCR (Detailed information about all 10 unigenes is given in Table S1.). Fruits from HL and WT at stage III were selected for RT-qPCR analysis, and $\beta$-actin was used as a reference gene in this study. As shown in Fig. 7, most of these DEGs showed a similar expression pattern according to RT-qPCR and RNA-seq results, which suggested that results from the transcriptome data are reliable.

\section{Discussion}

Hybrids often present phenotypes that surpass their parents in terms of growth and yield, a phenomenon known as "hybrid vigor" or "heterosis" [36]. Hybrid rice largely contributes to solve the problem of food for hundreds of millions of people all over the world due to heterosis effects on yield [37]. Recently, with the improvement of living standards, people pay more attention to health and tasty food. Euryale ferox Salisb. is considered as one suitable substitute for rice and wheat as it is widely distributed and has a waxy taste [38]. However, development of this crop faces many problems, such as high planting costs and low yield. To overcome these problems, we spent many years selecting dominant lines and crossbreeding them, until finally, a HL was developed. To our surprise, compared to the WT, the new hybrid exhibited significantly higher yield; in addition to which, it showed a number of advantageous traits, including non-prickly and heavily waxy seeds. After comparing this new HL with parent WT for yield traits, the size of kernel generated by the HL was much larger than that of WT, which revealed kernel size as a crucial factor in improving the yield of HL. Besides, the high yield of HL was also attributed to its heavier seeds, but seed weight primarily depends on seed size especially during the process before seed filling, indicating a crucial role for seed size in evaluation of yield of Euryale ferox Salisb. during the growth of its reproductive organs $[39,40]$. Seeds of aquatic plants often have higher moisture water, which induces a large error in seed weight [41]. As a consequence, seed size was taken as the main observation in this study to investigate functional genes and molecular mechanisms in Euryale ferox Salisb. 


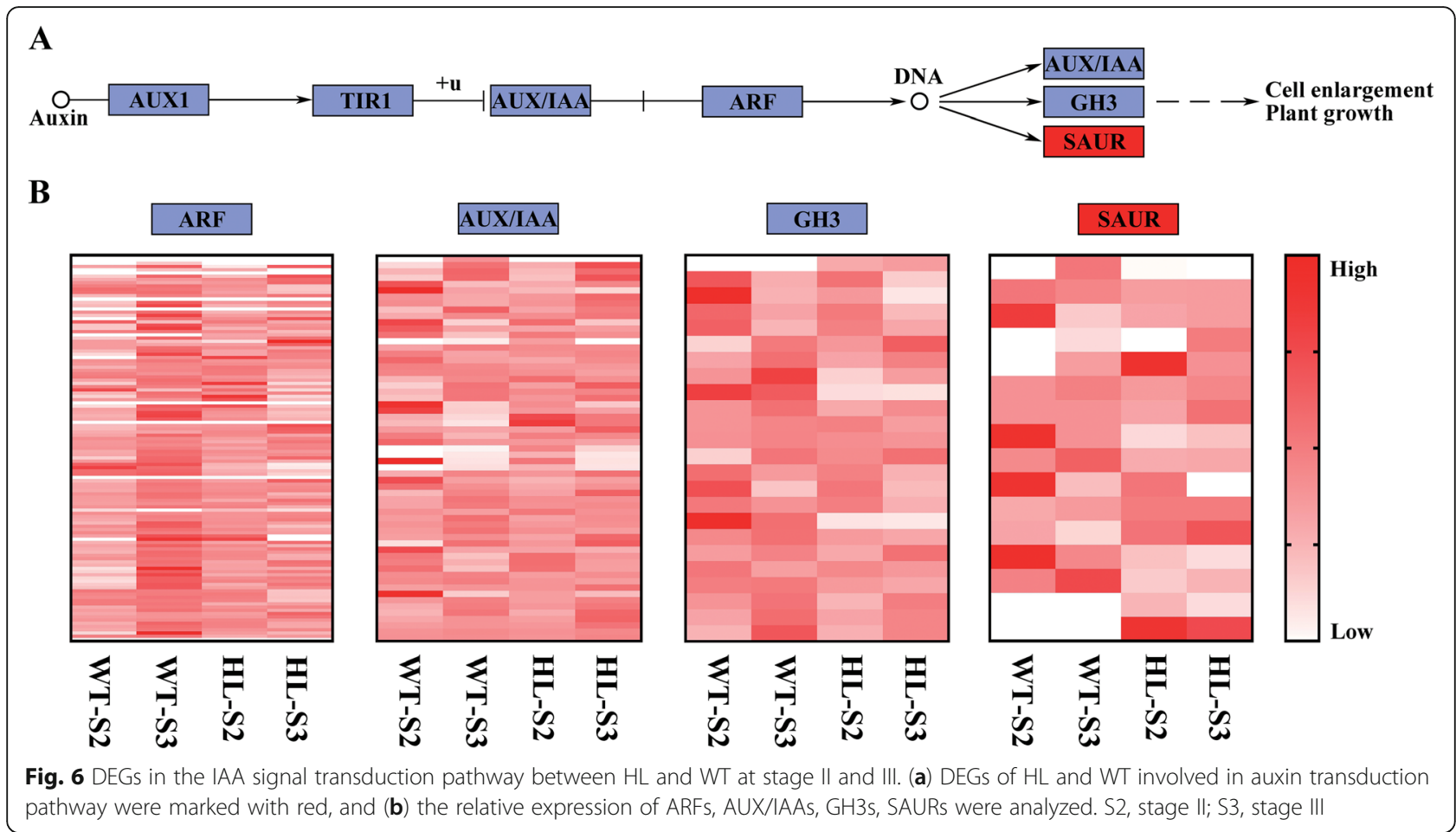

To establish a growth pattern of Euryale ferox Salisb., we divided the phases of fruit development into five stages according to average fruit growth above the water. Fruit growth rate is low over the first two stages, especially at stage II, which suggests that during these stages, vegetative and reproductive organs grow together. In contrast, over the remaining three stages, reproductive growth allows only fruit generation and growth [42]. The significant decrease in growth at stage $\mathrm{V}$ may be due to water loss in fruits during post-maturation [43]. The growth stage division of HL was based on rice studies and was critical for sample selection and time course studies of HL and WT. As expected, we observed a significant difference in fruit growth rate between the two lines under study. Factors that contributed to this difference became the focus of our study.

Although heterosis has been successfully applied to increase HL yield, the molecular mechanisms involved remained unknown [44]. As the difference in growth induced by heterosis can be largely revealed by transcriptome analysis, we performed RNA-seq analysis on the two lines $[45,46]$. Concentrations of auxin varied across different tissues, mediating distinct developmental outcomes and contributing to the functional diversity of auxin [47]. In our study, IAA content at the early stage did not show any difference between the two plant materials, whereas IAA localization at the same selected growth stages was different for the two lines. Plant tissues rapidly sense and respond to changes in auxin levels. These responses involve several major classes of auxin-responsive genes [48]. We hypothesized that, in WT, at this early stage pricks happen to constitute a protective natural barrier, and this process uses considerable amounts of IAA, whereas HL invests IAA only on developing ovaries and stamens. This also explains why the ovary of $\mathrm{HL}$ is somewhat larger than that of WT. We speculate that the elongation effect of IAA on the ovary in HL was influenced by the difference in IAA allocation pattern between HL and WT. In SE fruit, IAA signal mainly distributed near stamens, this is probably because SE grows faster than WT and HL, and with a longer period of flowering days. Furthermore, it is worth noting that this process is probably mediated by SAURs as, in the auxin transduction pathway, only this gene family was detected as a differentially expressed set of genes between the two lines at both growth stages. This hypothesis is supported by the fact that some SAURs promote cell expansion in plant tissues [49-51]. And many genes in IAA signal transduction pathway have been reported to affect the seed development $[52,53]$. However, there were also a few genes involved in $G H 3$ that were found upregulated in $\mathrm{HL}$ due to the large number of unigenes in GH3. These differences did not induce a significantly different expression of GH3s. Previously, the transcriptomic analysis in seed samples of Euryale ferox Salisb. suggested that PAL and P450-related genes promote the maturation of seed by affecting the phenylpropanoid biosynthesis, indicating a crucial role for genes or pathways in the promotion of development of Euryale ferox Salisb [54]. Differ from previous study, we used fruits of both WT and HL types for 


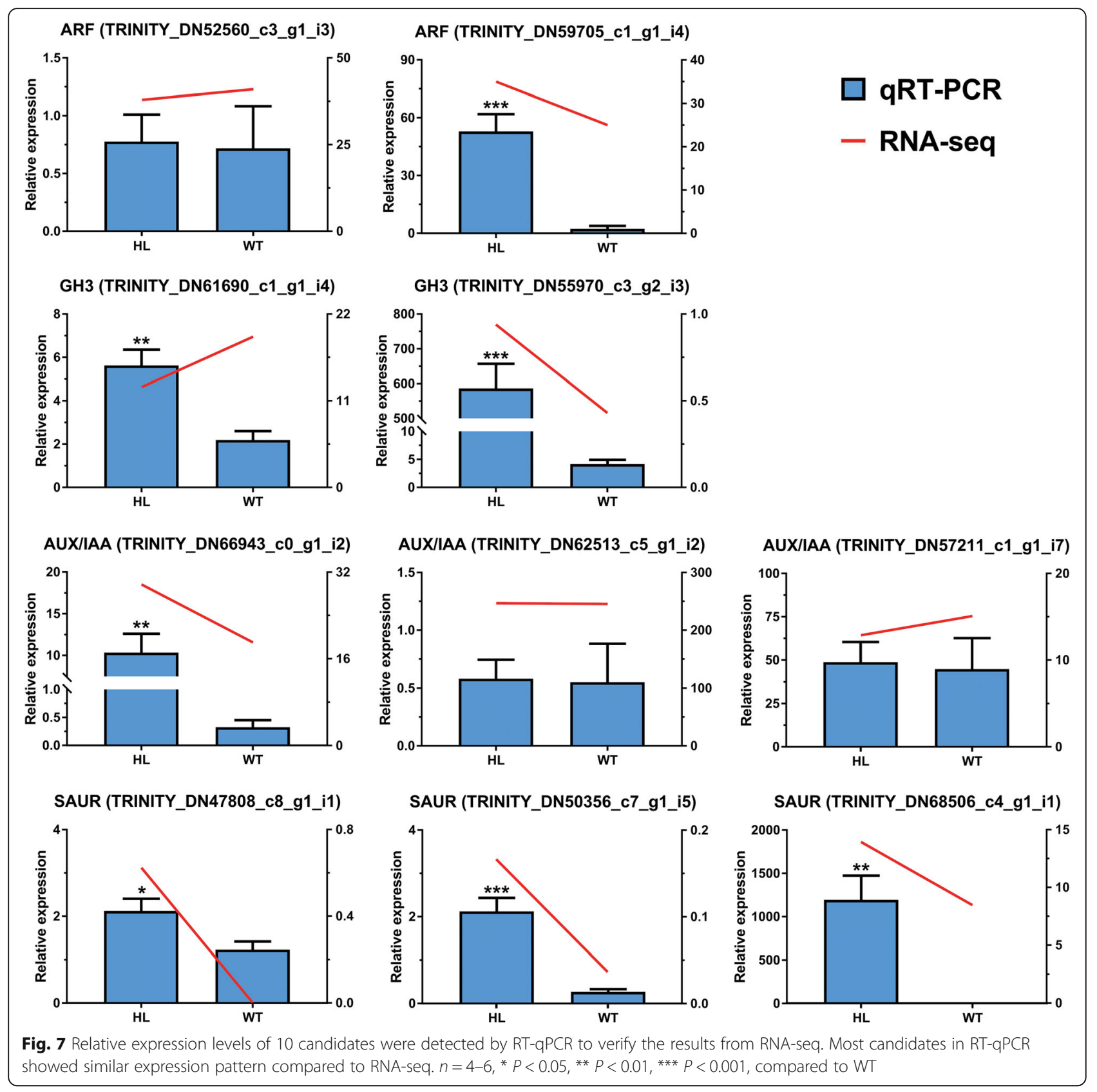

RNA-seq analysis and considered IAA-related genes as the potential function genes in seed development. Our study not only provided more complete reads and more precise annotations of unigenes in Euryale ferox Salisb. but also indicated a potential mechanism in promoting seed development.

Summary, though phenotypic observation, IAA detection and RNA-seq analysis, our results gradually revealed that SAURs potentially mediated the localization of IAA and thereby regulated seed size in HL. In addition to which, we also provided a preliminary investigation on functional genes and molecular mechanisms involved in seed formation of Euryale ferox Salisb.

\section{Conclusions}

Hybrid Euryale ferox Salisb. expressed significant heterosis, resulting in non-prickly, thin-coated, large seeds, which accounted for the significantly larger yield of HL than that of WT. Through the study, we found that some SAURs may act as a positive mediator of the auxin transduction pathway, thereby contributing to the observed larger seed. The gene functions of these SAURs 
in Euryale ferox Salisb., and the underlying mechanisms deserve further investigation.

\section{Methods}

Plant materials and growth conditions

Euryale ferox HL and its parents, North Gordon Euryale (E. ferox, WT) and South Gordon Euryale (E. ferox, SE), were used as plant materials in this study. Seeds of WT were originally obtained in 2001 from the wild (Gaoyou lake of Jiangsu province) and cultured in the experimental field of Jiangsu Seed and Seed Breeding Base, and seeds of SE were originally obtained from the wild (Suzhou of Jiangsu province) and also grown in the experimental field of Jiangsu Seed and Seed Breeding Base who provided permission for their use in this scientific research. HL (containing 3 lines) were constructed from WT as the female crossed with SE to generate $F_{1}$ hybrid in Gaoyou (E119 $\left.30^{\prime}, \mathrm{N} 32^{\circ} 58^{\prime}\right)$, Jiangsu province, China in 2015; later, $F_{1}$ was selected and self-pollinated to generate $F_{2}$ in Nanzha (E119 $\left.10^{\prime}, \mathrm{N} 33^{\circ} 18^{\prime}\right)$, Jiangsu province, China in 2016; and $F_{2}$ individuals were used to produce $\mathrm{F}_{3}$ by self-fertilization in Gaoyou (E119 $30^{\prime}$, N32 $58^{\prime}$ ), Jiangsu province, China in 2017; finally, $F_{3}$ generation and both of its parents were cultured in the experimental field of Jiangsu Seed and Seed Breeding Base (Gaoyou, Jiangsu province). All plant materials used in this study were supplied by the Jiangsu Seed and Seed Breeding Base. Collection of all samples completely complies with national and local legislation permission, and no specific permission was required for collecting these plants. The plant materials were formal identified by Professor Qinan Wu, and a voucher specimen of this material has been deposited in Nanjing University of Chines Medicine. HL and the WT were sown in a completely randomized block design with three replications in April 22, 2018. Each plot consisted of 160 individual lines, each separated $2 \mathrm{~m}$ from its neighboring lines. The two lines were selected in this study to measure phenotypic traits and conduct transcriptome analyses. The young fruits selected for sampling at the designated development stages were collected and stored at $-80^{\circ} \mathrm{C}$ for RNA-seq and RT-qPCR analysis. Seeds were collected at maturity on September 9, 2018; they were selected with 10 replicates for yield traits; additionally, each sample had at least three biological replications to minimize systematic errors.

\section{IAA quantification}

IAA was quantified by liquid chromatography-mass spectrometry (LC-MS), essentially as described [55]. Briefly, $50 \mathrm{mg}$ of frozen tissue was homogenized in $500 \mu \mathrm{L}$ of extraction buffer (isopropanol: double distilled water: acetic acid $=2: 1: 0.002, \mathrm{vol} / \mathrm{vol} / \mathrm{vol})$. After shaking on ice for $30 \mathrm{~min}, 1 \mathrm{~mL}$ dichloromethane was added to each sample and shaken for another $30 \mathrm{~min}$. Then, samples were centrifuged at $13,000 \mathrm{~g}$ for $5 \mathrm{~min}$ at $4{ }^{\circ} \mathrm{C}$; the lower phase was dried under a stream of nitrogen gas, and redissolved with methyl alcohol. Each sample solution was injected into the reverse-phase $\mathrm{C}_{18}$ HPLC column $(2.1 \mathrm{~mm} \times 100 \mathrm{~mm}, 2.7 \mu \mathrm{m}$, Agilent, USA) for LCMS (Shimadzu LC-30 ultrahigh performance liquid chromatography system, Shimadzu, Japan; AB Sciex QTRAP 5500, SCIEX, USA) analysis. An IAA standard $(\geq 98 \%, 45,533$, Sigma, USA) was used as the external standard.

\section{Immunofluorescence staining}

Immunofluorescence staining for IAA was performed on sections of fruits as follows: Briefly, $60 \mu \mathrm{m}$ fruit sections were cut using a cryotome (CM1950, Leica Microsystems, Germany) and affixed onto glass slides, followed by dehydration in ascending and descending ethanol solutions. After washing three times for $10 \mathrm{~min}$ in phosphate buffer saline (PBS) buffer with $0.1 \%(\mathrm{v} / \mathrm{v})$ Tween 20 , sections were pre-treated with $5 \%(\mathrm{w} / \mathrm{v})$ bovine serum albumin in PBS for $1 \mathrm{~h}$ at room temperature to reduce non-specific binding, followed by incubating overnight with anti-IAA antibody (1:200, AS09 421, Agrisera, Sweden) at $4{ }^{\circ} \mathrm{C}$. After washing three times with $0.1 \%(\mathrm{v} / \mathrm{v})$ Tween 20 in PBS buffer for $10 \mathrm{~min}$, sections were incubated with Alexa Fluor 594 (1:200, conjugated Goat Anti-Rabbit IgG $(\mathrm{H}+\mathrm{L})$, SA00006-4, Proteintech Group, USA) as a secondary antibody for 1 $\mathrm{h}$ in darkness at room temperature. Samples were washed three times for 10 min with $0.1 \%$ (v/v) Tween 20 in PBS. Sections were imaged using a Carl Zeiss Axio Scope fluorescence microscope (Gottingen, Germany). Samples incubated with PBS instead of primary antibodies were used as negative controls, and there was no unspecific binding of the secondary antibody in this study.

\section{RNA extraction, CDNA library preparation and sequencing} Before total RNA was extracted from samples, 12 frozen tissue samples were fully grinded separately under liquid nitrogen. RNA quality was determined by Agilent 2100 BisAnalyzer (J06-02, Agilent, USA), and samples with both $\mathrm{RIN} \geq 7$ and $\frac{28 S}{18 \mathrm{~s}} \geq 1.5$ were included for RNA-seq analysis. RNA concentration was determined using a Qubit RNA BR Assay Kit (Q10211, Invitrogen, USA).

To construct the cDNA libraries, $5 \mu \mathrm{g}$ total RNA per sample was used as the template. The NEBNext Ultra RNA Library Prep Kit for Illumina (E7530S, NEB, USA) was used to generate a series of corresponding libraries, and index codes were applied to each sample. Briefly, mRNA was purified from total RNA using oligod (T) beads. RNA fragmentation was carried out at high 
temperature in the presence of divalent cations in NEBNext First-Strand Synthesis Reaction Buffer (5X). Firststrand cDNA was synthesized using random hexamer primers and M-MLV Reverse Transcriptase. Secondstrand cDNA synthesis was subsequently performed using DNA polymerase I, RNase $\mathrm{H}, \mathrm{dNTP}$ and buffer. Then, the cDNA fragments were purified, end repaired, and poly (A) added, followed by adapter ligation. The selected ligation product was amplified and, subsequently, sequenced with Illumina HiSeq TM X10 by Gene Denovo Biotechnology Co. (Beijing, China).

\section{Identification of differentially expressed mRNAs}

After high-throughput sequencing, raw reads with too many unknown bases $(>5 \%)$ or low-quality bases $(>30 \%$ of the bases with a quality score $\leq 20$ ) were excluded to obtain clean reads. The remaining clean reads from each sample were then assembled by Trinity ${ }^{3}$ with de Bruijn graph.

The unigene sequences were annotated using the following public databases: Universal Protein (Uniprot), evolutionary genealogy of genes (Eggnog), Pfam protein domain database (Pfam), signal peptide server (SignalP), Hidden Markov Model-based transmembrane protein database (TMhmm), Kyoto Encyclopedia of Genes and Genomes (KEGG), and Gene Ontology (GO). The unigene sequences were aligned using BLASTx with an evalue of $<10^{-5}$, and the annotations were only saved when the "-max_target_seq" was "1".

Differential expression was estimated and tested using the RSEM ${ }^{12}$ software with the EM method. We quantified gene expression levels in terms of Fragments Per Kilo bases per Million (FPKM), calculated the false discovery rate (FDR), and estimated the fold change (FC) and $\log _{2}$ values of FC. Transcripts that exhibited an FDR $\leq 0.01$ and an estimated absolute $\left|\log _{2}(\mathrm{FC})\right| \geq 1$ were considered to be significantly differentially expressed.

\section{Quantitative real-time PCR (RT-qPCR) analysis}

RNA was extracted using TRIzol (T9424, Sigma, USA) and $1 \mu \mathrm{g}$ used for cDNA synthesis with the PrimeScript RT Master Mix kit (RR036A, TaKaRa, China). RT-qPCR was performed with a QuanStudio 3 instrument (Applied Biosystems, USA), using $5 \mu \mathrm{L}$ cDNA, NovoStart SYBR qPCR SuperMix Plus (E096-01B, Novoprotein, China), and $0.2 \mu \mathrm{L}$ each of two gene-specific primers (Table S1) in a final volume of $20 \mu \mathrm{L}$. The thermocycling regime consisted of $2 \mathrm{~min}$ at $50^{\circ} \mathrm{C}, 10 \mathrm{~min}$ at $95^{\circ} \mathrm{C}$, followed by 40 cycles of $15 \mathrm{~s}$ at $95^{\circ} \mathrm{C}, 1 \mathrm{~min}$ at $55^{\circ} \mathrm{C}$, and $30 \mathrm{~s}$ at $72^{\circ} \mathrm{C}$. Disassociation curves verified amplification of a single product. The relative expression of each gene was analyzed using the comparative $\mathrm{Ct}(\Delta \Delta \mathrm{Ct})$ method with $\beta$-actin as a reference gene for normalization. The reference gene was selected according to previous studies [56]. Briefly, gene-specific primers were designed by Primer Premier 5 and checked by melting curve after amplification. The expression stability of each candidate reference gene was compared and ranked by BestKeeper software according to the threshold cycle $(\mathrm{Ct})$ values. Reference genes with a standard deviation value below 1 were considered as stably expressed in this study, and a smaller coefficient of variation indicates a more stable reference gene. Each amplification reaction was performed in triplicate.

\section{Data analysis}

All data were expressed as the mean \pm standard error of the mean. Differences between two groups were analyzed using $t$-test and $P<0.05$ was considered to indicate a statistically significant difference. Statistical analysis was performed with GraphPad Prism 6.0 software (GraphPad Software, Inc.).

\section{Supplementary information}

Supplementary information accompanies this paper at https://doi.org/10. 1186/s12870-020-02504-2.

Additional file 1: Figure S1. The development stage division of fruits. Additional file 2: Figure S2. Width of fruits in $H L$ and WT.

Additional file 3: Figure S3. Concentration and distribution of IAA at stage II.

Additional file 4: Figure S4. Distribution of IAA in fruits of SE.

Additional file 5: Table S1. Detail information of Unigenes.

\section{Abbreviations}

HL: Hybrid line; WT: North Gordon Euryale (wild type); IAA: Indole-3-acetic acid; TIR1/AFB: Transport Inhibitor Response1/Auxin Signaling F-box Protein; ARF: Auxin Response Factor; SAUR: Small auxin up RNA; GH3: Glycoside Hydrolase 3; AUXIIAA: Auxin/Indole-3-Acetic Acid; DEG: Differentially expressed genes; LC-MS: Liquid chromatography-mass spectrometry; PBS: Phosphate buffer saline; Uniprot: Universal Protein database; Eggnog: Evolutionary genealogy of genes database; Pfam: Pfam protein domain database; SignalP: Signal peptide server database; TMhmm: Hidden Markov Modelbased transmembrane protein database; KEGG: Kyoto Encyclopedia of Genes and Genomes database; GO: Gene Ontology database; FPKM: Fragments Per Kilo bases per Million; FDR: Calculated the false discovery rate; FC: Fold change; RT-qPCR: Quantitative real-time PCR; $\Delta \Delta$ Ct: Comparative Ct

\section{Acknowledgements}

We also thank Editage (www.Editage.cn) for English language editing.

\section{Authors' contributions}

QW and ZH designed and oversaw the research. ZH, ZJ and KB performed the research. QW and $\mathrm{HD}$ analyzed the results and wrote the manuscript. YZ and SZ provided helpful suggestions during the data analysis. ZH and QW provided suggestions during the article writing. KB provided help with cotton field management. All authors read and approved the final manuscript.

\section{Funding}

The authors gratefully acknowledge the financial support of Chinese National Natural Science Foundation (81773854); The Program of Collaborative Innovation Center of Chinese Medicinal Material Resources Industrialization of Jiangsu Province (2016); Postgraduate Research \&Practice Innovation Program of Jiangsu Province (KYCX19_1247; KYCX19_1333). 


\section{Availability of data and materials}

The datasets used and/or analyzed in the current study are available from the corresponding author upon reasonable request. The role of the funding body in the design of the study and collection, analysis, and interpretation of data and in writing the article should be declared in this request. All Illumina Sequencing data have been deposited in NCBI Sequence Read Archive (SRA) under accession number: PRJNA628746 (https://www.ncbi.nlm.nih.gov/ bioproject/PRJNA628746).

\section{Ethics approval and consent to participate}

Not applicable.

\section{Consent for publication}

Not applicable.

\section{Competing interests}

The authors declare that they have no competing interests.

Received: 5 November 2019 Accepted: 18 June 2020

Published online: 03 July 2020

\section{References}

1. Kumar N, Shikha D, Kumari S, Choudhary BK, Kumar L, Singh IS. SSR-based DNA fingerprinting and diversity assessment among Indian Germplasm of Euryale ferox: an aquatic underutilized and neglected food crop. Appl Biochem Biotechnol. 2018:185(1):34-41.

2. Jha V, Shalini R, Kumari A, Jha P, Sah NK. Aquacultural, nutritional and therapeutic biology of delicious seeds of Euryale ferox Salisb. : A Minireview. Curr Pharm Biotechnol. 2018;19(7):545-55.

3. Ahmed D, Kumar V, Verma A, Shukla GS, Sharma M. Antidiabetic, antioxidant, antihyperlipidemic effect of extract of Euryale ferox salisb. with enhanced histopathology of pancreas, liver and kidney in streptozotocin induced diabetic rats. SpringerPlus. 2015:4:315.

4. Wu CY, Wang H, He XX, Wu DW, Yue W, Wu QN. The hypoglycemic and antioxidant effects of polysaccharides from the petioles and pedicels of Euryale ferox Salisb. On alloxan-induced hyperglycemic mice. Food Funct. 2017:8(10):3803-13.

5. Huang Z, Huang $X$, Wang $Q$, Jiang $R$, Sun $G, X u Y$, et al. Extract of Euryale ferox Salisb exerts antidepressant effects and regulates autophagy through the adenosine monophosphate-activated protein kinase-UNC-51-like kinase 1 pathway. IUBMB Life. 2018;70(4):300-9.

6. Xu X, Wu YF, Zhang Y, Wu P, Li L. The amylopectin characteristics and the GeSBE1 gene expression in seed kernels of Euryale ferox Salisb. Chin I Trop Crops. 2019:40(3):514-21.

7. Kumar R, Khurana A, Sharma AK. Role of plant hormones and their interplay in development and ripening of fleshy fruits. J Exp Bot. 2014;65(16):4561-75.

8. Kurepin LV, Zaman M, Pharis RP. Phytohormonal basis for the plant growth promoting action of naturally occurring biostimulators. J Sci Food Agric. 2014;94(9):1715-22.

9. Alabadi D, Blazquez MA, Carbonell J, Ferrandiz C, Perez-Amador MA. Instructive roles for hormones in plant development. Int J Dev Biol. 2009; 53(8-10):1597-608.

10. Korasick DA, Enders TA, Strader LC. Auxin biosynthesis and storage forms. J Exp Bot. 2013;64(9):2541-55.

11. Zhao Y. Auxin biosynthesis and its role in plant development. Annu Rev Plant Biol. 2010;61:49-64.

12. Salehin M, Bagchi R, Estelle M. SCFTIR1/AFB-based auxin perception: mechanism and role in plant growth and development. Plant Cell. 2015;27(1):9-19.

13. Adamowski M, Friml J. PIN-dependent auxin transport: action, regulation, and evolution. Plant Cell. 2015;27(1):20-32.

14. Lakehal A, Chaabouni S, Cavel E, Le Hir R, Ranjan A, Raneshan Z, et al. A molecular framework for the control of adventitious rooting by TIR1/AFB2-auX/ IAA-dependent Auxin signaling in Arabidopsis. Mol Plant. 2019;12(11):1499-514.

15. Ishimaru K, Hirotsu N, Madoka Y, Murakami N, Hara N, Onodera H, et al. Loss of function of the IAA-glucose hydrolase gene TGW6 enhances rice grain weight and increases yield. Nat Genet. 2013;45(6):707-11.

16. Kuang JF, Wu JY, Zhong HY, Li CQ, Chen JY, Lu WJ, et al. Carbohydrate stress affecting fruitlet abscission and expression of genes related to auxin signal transduction pathway in litchi. Int J Mol Sci. 2012;13(12):16084-103.

17. van den Berg T, Ten Tusscher KH. Auxin Information Processing; Partners and Interactions beyond the Usual Suspects. Int J Mol Sci. 2017;18(12):2585.
18. Hagen G. Auxin signal transduction. Essays Biochem. 2015;58:1-12.

19. Lavy M, Estelle M. Mechanisms of auxin signaling. Development. 2016; 143(18):3226-9.

20. Leyser O. Auxin signaling. Plant Physiol. 2018;176(1):465-79.

21. Stortenbeker N, Bemer M. The SAUR gene family: the plant's toolbox for adaptation of growth and development. J Exp Bot. 2019;70(1):17-27.

22. van Mourik H, van Dijk ADJ, Stortenbeker N, Angenent GC, Bemer M. Divergent regulation of Arabidopsis SAUR genes: a focus on the SAUR10clade. BMC Plant Biol. 2017;17(1):245.

23. Bemer M, van Mourik H, Muino JM, Ferrandiz C, Kaufmann K, Angenent GC. FRUITFULL controls SAUR10 expression and regulates Arabidopsis growth and architecture. J Exp Bot. 2017;68(13):3391-403.

24. Chae K, Isaacs CG, Reeves PH, Maloney GS, Muday GK, Nagpal P, et al. Arabidopsis SMALL AUXIN UP RNA63 promotes hypocotyl and stamen filament elongation. Plant J. 2012;71(4):684-97.

25. Kant S, Bi YM, Zhu T, Rothstein SJ. SAUR39, a small auxin-up RNA gene, acts as a negative regulator of auxin synthesis and transport in rice. Plant Physiol. 2009;151(2):691-701.

26. Kant $\mathrm{S}$, Rothstein $\mathrm{S}$. Auxin-responsive SAUR39 gene modulates auxin level in rice. Plant Signal Behav. 2009;4(12):1174-5.

27. Zhang W, Tan L, Sun H, Zhao X, Liu F, Cai H, et al. Natural variations at TIG1 encoding a TCP transcription factor contribute to plant architecture domestication in Rice. Mol Plant. 2019:12(8):1075-89.

28. Imanishi A, Imanishi J. Seed dormancy and germination traits of an endangered aquatic plant species, Euryale ferox Salisb. (Nymphaeaceae). Aquat Bot. 2014;119:80-3.

29. Jha V, Kargupta AN, Dutta RN, Jha UN, Mishra RK, Saraswati KC. Utilization and conservation of Euryale ferox Salisbury in Mathila (North Bihar), India. Aquat Bot. 1991;39(3):295-314.

30. Zuo J, Li J. Molecular genetic dissection of quantitative trait loci regulating rice grain size. Annu Rev Genet. 2014;48:99-118.

31. Zhang C, Yin Y, Zhang A, Lu Q, Wen X, Zhu Z, et al. Comparative proteomic study reveals dynamic proteome changes between superhybrid rice LYP9 and its parents at different developmental stages. J Plant Physiol. 2012; 169(4):387-98.

32. Yang J, Luo D, Yang B, Frommer WB, Eom JS. SWEET11 and 15 as key players in seed filling in rice. New Phytol. 2018;218(2):604-15.

33. Ljung K, Bhalerao RP, Sandberg G. Sites and homeostatic control of auxin biosynthesis in Arabidopsis during vegetative growth. Plant J. 2001;28(4): 465-74.

34. Nishimura T, Toyooka K, Sato M, Matsumoto S, Lucas MM, Strnad M, et al. Immunohistochemical observation of indole-3-acetic acid at the IAA synthetic maize coleoptile tips. Plant Signal Behav. 2011;6(12):2013-22.

35. Tagore S, Chowdhury N, De RK. Analyzing methods for path mining with applications in metabolomics. Gene. 2014;534(2):125-38.

36. Huang X, Yang S, Gong J, Zhao Q, Feng Q, Zhan Q, et al. Genomic architecture of heterosis for yield traits in rice. Nature. 2016;537(7622):629-33.

37. Li D, Huang Z, Song $S$, Xin $Y$, Mao D, Lv Q, et al. Integrated analysis of phenome, genome, and transcriptome of hybrid rice uncovered multiple heterosis-related loci for yield increase. Proc Natl Acad Sci U S A. 2016; 113(41):E6026-E35.

38. Puste AM, Sarkar PK, Das DK. Balanced nitrogen economy as a flexible strategy on yield stabilizing and quality of aquatic food crops in wetland ecosystem. Sci China C Life Sci. 2005;48 Spec No:980-7.

39. Arshad MS, Faroog M, Asch F, Krishna JSV, Prasad PW, Siddique KHM Thermal stress impacts reproductive development and grain yield in rice. Plant Physiol Biochem. 2017;115:57-72.

40. Benincasa P, Reale L, Tedeschini E, Ferri V, Cerri M, Ghitarrini S, et al. The relationship between grain and ovary size in wheat: an analysis of contrasting grain weight cultivars under different growing conditions. Field Crop Res. 2017:210:175-82.

41. Chen C, Fu W, Chang Q, Zheng B, Zhang Y, Zeng H. Moisture distribution model describes the effect of water content on the structural properties of lotus seed resistant starch. Food Chem. 2019:286:449-58.

42. Miura K, Ikeda M, Matsubara A, Song XJ, Ito M, Asano K, et al. OsSPL14 promotes panicle branching and higher grain productivity in rice. Nat Genet. 2010;42(6):545-9.

43. Sheehy JE, Mitchell PL, Ferrer AB. Bi-phasic growth patterns in rice. Ann Bot. 2004;94(6):811-7.

44. Huyen NT. Another explanation for the cause of heterosis phenomenon. Genet. 2016;95(4):1065-72. 
45. Yang $M$, Wang $X$, Ren $D$, Huang $H, X u M, H e ~ G$, et al. Genomic architecture of biomass heterosis in Arabidopsis. Proc Natl Acad Sci U S A. 2017;114(30): 8101-6.

46. Shao L, Xing F, Xu C, Zhang Q, Che J, Wang X, et al. Patterns of genomewide allele-specific expression in hybrid rice and the implications on the genetic basis of heterosis. Proc Natl Acad Sci U S A. 2019;116(12):5653-8.

47. Cao M, Chen R, Li P, Yu Y, Zheng R, Ge D, et al. TMK1-mediated auxin signalling regulates differential growth of the apical hook. Nature. 2019; 568(7751):240-3.

48. Luo J, Zhou JJ, Zhang JZ. Aux/IAA Gene Family in Plants: Molecular Structure, Regulation, and Function. Int J Mol Sci. 2018;19(1).

49. Spartz AK, Ren H, Park MY, Grandt KN, Lee SH, Murphy AS, et al. SAUR inhibition of PP2C-D phosphatases activates plasma membrane H+-ATPases to promote cell expansion in Arabidopsis. Plant Cell. 2014;26(5):2129-42.

50. Sun N, Wang J, Gao Z, Dong J, He H, Terzaghi W, et al. Arabidopsis SAURs are critical for differential light regulation of the development of various organs. Proc Natl Acad Sci U S A. 2016;113(21):6071-6.

51. Favero DS, Le KN, Neff MM. Brassinosteroid signaling converges with SUPPRESSOR OF PHYTOCHROME B4-\#3 to influence the expression of SMALL AUXIN UP RNA genes and hypocotyl growth. Plant J. 2017;89(6): 1133-45.

52. Agarwal P, Singh PC, Chaudhry V, Shirke PA, Chakrabarty D, Farooqui A, et al. PGPR-induced OsASR6 improves plant growth and yield by altering root auxin sensitivity and the xylem structure in transgenic Arabidopsis thaliana. J Plant Physiol. 2019;240.

53. Zhou L, Hou HZ, Yang T, Lian YK, Sun Y, Bian ZY, et al. Exogenous hydrogen peroxide inhibits primary root gravitropism by regulating auxin distribution during Arabidopsis seed germination. Plant Physiol Biochem. 2018;128:126-33.

54. Liu X, He Z, Yin Y, Xu X, Wu W, Li L. Transcriptome sequencing and analysis during seed growth and development in Euryale ferox Salisb. BMC Genomics. 2018;19(1):343.

55. Pan X, Welti R, Wang X. Quantitative analysis of major plant hormones in crude plant extracts by high-performance liquid chromatography-mass spectrometry. Nat Protoc. 2010;5(6):986-92.

56. Nong Q, Yang Y, Zhang M, Zhang M, Chen J, Jian S, et al. RNA-seq-based selection of reference genes for RT-qPCR analysis of pitaya. FEBS Open Bio. 2019;9(8):1403-12

\section{Publisher's Note}

Springer Nature remains neutral with regard to jurisdictional claims in published maps and institutional affiliations.

Ready to submit your research? Choose BMC and benefit from:

- fast, convenient online submission

- thorough peer review by experienced researchers in your field

- rapid publication on acceptance

- support for research data, including large and complex data types

- gold Open Access which fosters wider collaboration and increased citations

- maximum visibility for your research: over $100 \mathrm{M}$ website views per year

At BMC, research is always in progress.

Learn more biomedcentral.com/submissions 